\title{
Harrington Rod Instrumentation: A Cause of Brown-Sequard Syndrome
}

\author{
C.B. Van Orman and H.Z. Darwish
}

\begin{abstract}
We describe a child with Brown-Sequard syndrome beginning at C8-T1, immediately following Harrington rod instrumentation for idiopathic scoliosis. We believe this is most likely related to either a common variation of the branches of the anterior spinal artery to one side or due to rupture of the ipsilateral spinal vein. This possible complication should be considered in patients undergoing Harrington rod instrumentation.
\end{abstract}

\begin{abstract}
RÉSUMÉ: L'implantation d'une tige de Harrington comme cause du syndrome de Brown-Séquard. Nous décrivons le cas d'un enfant présentant un syndrome de Brown-Séquard commençant à C8-D1, immédiatement après l'implantation chirurgicale d'une tige de Harrington pour scoliose idiopathique. Nous croyons que ce phénomène est vraisemblablement relié à une variante fréquente des branches de l'artère spinale antérieure d'un côté ou due à la rupture de la veine spinale ipsilatérale. Ce syndrome doit être envisagé comme une complication possible chez les patients qui subissent une chirurgie de ce type.
\end{abstract}

Can. J. Neurol. Sci. 1988; 15:44-46

Idiopathic adolescent scoliosis occurs in approximately 1.8 percent of the general population but in 3.9 percent of white girls.' The patient's age, as determined by skeletal maturity, and the degree of the curve at the time of diagnosis influence the choice of such management options as bracing, surface electrical stimulation or surgery. ${ }^{2}$ At present, the Harrington technique is the most widely used surgical technique and is generally reserved for curves over 42 to 45 degrees which have been shown to be progressive. ${ }^{3}$ The reported incidence of acute neurological complications following scoliosis surgery has ranged up to $\mathbf{1 7}$ percent, with a survey from the Scoliosis Research Society recording an incidence of 0.72 percent (fifty-seven of 7,885 patients). ${ }^{4}$ Although transient sensory changes and other minor neurological complications occur, the most frequent and severe complication is paraplegia. This is a report of a child who developed Brown-Sequard syndrome following Harrington rod instrumentation.

\section{HISTORY}

A 131/2-year-old girl was assessed for a three month history of an asymptomatic rotational spine deformity. Examination at that time revealed a clinically mobile curve without kyphosis. Plain roentgenograms confirmed a 54 degree curve at D5-D12, convex to the right, with wedging of D7, D8 and D9. She was placed in a Milwaukee brace. Within one year the curve had progressed to 60 degrees with a grade I rotatory component.
Accordingly, the decision was made to proceed to operative intervention. At age 14 years 9 months she underwent a D2 to D12 Harrington rod instrumentation and fusion without prior traction. The operation was performed in a standard manner without complication. Intraoperatively, the systemic blood pressure rose briefly from $110 / 50$ to $150 / 70$ and then stabilized at $90 / 45$. Operative blood loss was estimated at $1500 \mathrm{ml}$; the patient received $750 \mathrm{ml}$ packed red cells and $3900 \mathrm{ml}$ of crystalloid intraoperatively. Although neither intraoperative somatosensory evoked potential monitoring nor an intraoperative wake up test were employed, the patient was noted in the recovery room to have "full motor control over both legs upon verbal command".

Seven hours after the operation, the patient complained of right upper arm and forearm discomfort and tingling. Decreased pain sensitivity on the left side of the body was noted in the first 24 hours postoperatively. The right hand grip was weak, and the patient was unable to fully extend the metacarpophalangeal joints of the fourth and fifth digits. Mild right leg weakness was also noted. The pupils were asymmetric with a miotic right pupil and an "edematous" right eyelid. Neurological consultation was not obtained in hospital, and a complete neurological examination was not documented at this time. The patient subsequently reported that there were no changes in symptomatology at the time of discharge nine days postoperatively.

Three months later, the patient still had left thermoanaesthesia, righthand weakness and an abnormal gait. The pupillary asym* 
metry had resolved. Spine roentgenograms showed a right scoliosis of 61 degrees with dislodgement of the inferior and both superior compression hooks and the superior distraction hook. Neurologic consultation was sought at this time, and examination demonstrated atrophy of the intrinsic hand muscles on the right. There was weakness (MRC grade $3 / 5$ power) of the right hand muscles including the lumbricals, first dorsal interosseous, abductor pollicis brevis, opponens pollicis, extensor and abductor digiti quinti. Muscle power was normal in the left upper extremity and in muscle groups innervated by $\mathrm{C} 7$ and higher semental spinal cord levels on the right. Motor power in the lower extremities was normal. A mild increase in tone and hyperreflexia in both the upper and lower extremities on the right side were elicited. There was a Babinski sign present on the right. Sensory examination revealed mild impairment of vibration sense in the right lower limb. There was complete analgesia and thermoanaesthesia in the $\mathrm{T} 1$ and $\mathrm{T} 2$ dermatomes on the right side with similar complete sensory deficit below T3 on the left side. These deficits were unchanged on re-examination five months later.

Limited electrophysiologic studies were completed three months after the operation. Normal distal motor and sensory conduction velocities in the right ulnar and median nerves below the elbow were documented. EMG confirmed denervation in the right abductor pollicis brevis with the presence of $2+$ fibrillation, $2+$ fasciculation, bizarre motor unit potentials, rare polyphasic potentials and absence of a recruiting pattern.

Table 1: Conditions Carrying an Increased Risk of Neurological Injury Following Scoliosis Surgery

1. Kyphosis

2. Congenital scoliosis

3. Scoliosis of severe degree

4. Pre-existing neurological deficit

5. Neurological deficit acquired in skeletal traction

(Adapted from MacEwen GD et al) ${ }^{4}$

Table 2: Procedures Carrying an Increased Risk of Neurological Injury Following Scoliosis Surgery

1. Skeletal traction

2. Spinal osteotomy

3. Harrington instrumentation with:

- congenital scoliosis without pre-operative traction

- correction greater than pre-operative traction

4. Segmental spinal instrumentation

(Adapted from MacEwen GD et al ${ }^{4}$ and Wilber RG et al) ${ }^{5}$
EMG of the right deltoid muscle was normal. A spinal CT failed because of artifact from the Harrington rods. Tomograms of the spine were normal at $D_{1}, D_{2}$ and $D_{3}$.

\section{Discussion}

This patient developed Brown-Sequard syndrome within hours of awakening from Harrington rod instrumentation for idiopathic scoliosis. Certain underlying conditions (Table 1 ) and particular management procedures (Table 2) have been noted to carry an increased risk of neurological injury. None of the previously identified risk factors were present in this patient.

A literature review of the etiology of Brown-Sequard syndrome does not identify Harrington rod instrumentation as a cause. On the other hand, a review of complications of scoliosis surgery indicates two poorly documented cases of "BrownSequard syndrome" (Table 3). ${ }^{5}$ The most common neurological complications of scoliosis surgery are transient sensory changes. Occasional motor deficits are also encountered, ranging from mild monoparesis to a complete paraplegia.

Though the intraoperative "wake up" test during scoliosis surgery may be useful in detecting paraplegia if it were to develop immediately, it may not identify the development of Brown-Sequard syndrome. ${ }^{6}$ In one patient described by Wilber et al, the wake up test showed some changes but bilateral movement was present before the end of the procedure. ${ }^{5}$ Most centres employ intraoperative somatosensory evoked potential monitoring. Intraoperative SEP changes did occur in the two patients described by Wilber et al. ${ }^{s}$ In both, however, the change in latency and amplitude improved before the end of the procedure.

Recently, documented cases of post operative neurological deficits have been described in patients who had "normal" somatosensory evoked potentials during intraoperative monitoring. ${ }^{7}$ SEP monitoring should be used with a clear awareness of its limitations, until better methods for intraoperative assessment during spinal surgery become available.

The postulated mechanisms of spinal cord injury following scoliosis surgery include: (1) Direct trauma to the cord; (2) Direct trauma to radiculomedullary arteries; (3) The adverse effects of mechanical tension, with a) undue traction on vessels causing ischemia and possibly minor hemorrhages; or, b) overstretching of the tracts and other neural components of the spinal cord.

The pathogenetic mechanism of Brown-Sequard syndrome seen in this patient, and perhaps in the two other cases cited in the literature is not clear. Direct intraoperative cord or spinal

Table 3

\begin{tabular}{|c|c|c|c|c|c|}
\hline & (Ref) & Age/Sex & $\begin{array}{c}\text { Type of } \\
\text { Scoliosis/Degree }\end{array}$ & Risk Factors & Neurologic Deficit \\
\hline 1. & (5) & 16 & Idiopathic $\left(45^{\circ}\right)$ & SSI* $^{*}$ & $\begin{array}{l}\text { - "BSS** involving both lower extremities", } \\
\text { "mild residual weakness' right LE unchanged at } 21 / 2 \text { years. }\end{array}$ \\
\hline 3. & (This Report) & 14 & Idiopathic $\left(60^{\circ}\right)$ & 0 & - BSS beginning at $\mathrm{C}_{8}-\mathrm{T}_{1}$. Persistent at 5 months. \\
\hline
\end{tabular}

*SSI = Segmental Spinal Instrumentation

${ }^{* *}$ BSS $=$ Brown-Sequard Syndrome 
artery injury cannot be excluded but the operation went smoothly with no recognized problems. Moreover, direct trauma to the cord during scoliosis surgery is very uncommon having been described in patients with absence of posterior osseous elements, in patients undergoing an anterior approach to the spine ${ }^{4}$ and in patients with segmental spinal instrumentation. ${ }^{8}$ None of these risk factors were present in this patient.

Alternatively, the mechanism of injury may have been vascular embarrassment of the spinal cord. The normal anatomy of the spinal cord vessels is such that the anterior spinal circulation has a limited ability to compensate for vascular occlusions, because it receives arterial feeders at very few levels and because it has a single channel, the anterior spinal artery, running longitudinally for a considerable distance without any additional collateral supply. In fact, in one study, ${ }^{9} 25 \%$ of candidates for corrective surgery for scoliosis had only one arterial feeder to the anterior spinal artery between T4 and L2. Dolan et al studied the effect of distraction on spinal cord blood flow using an animal model. ${ }^{10}$ Severe ischemia of the spinal cord caudal to the site of maximum distraction was seen even in the cats in the "short stretch" group. Furthermore, pathological examination of the cord revealed no hemorrhages or lacerations. The amount of distraction necessary to cause ischemic damage to the cord may be much lower in such pathological states as scoliosis compared to the normal spine. Hilal and Keim have shown that some patients with significant scoliosis have very poor spinal cord perfusion on angiography. ${ }^{9}$ However, direct, selective trauma to a radiculomedullary artery or ischemic cord injury induced by distraction generally produces a bilateral, symmetric neurologic deficit, particularly paraplegia.

Clearly, in this patient, the pattern of injury with greater contralateral lateral spinothalamic loss with minor ipsilateral corticospinal tract and dorsal column deficits, is consistent with a lesion affecting the anterior two-thirds of only the right side of the cord. Disruption of blood flow to that region could occur through several mechanisms. It is conceivable that if excessive distracting force is applied, the displacement of the cord in an upward direction and toward the center of the spinal canal could result in a significant stretching of the anterior segmental radiculomedullary arteries, particularly the shorter ones arising from the concave side of the scoliosis. However, in this patient the concavity was on the left side and the lesion occurred on the right. Alternatively this may be explained on the basis of obstruction of the sulcocommissural arterial branch to one side of the cord, a mechanism previously described by Wells. ${ }^{11}$ Additional alternatives may be the persistence of an anomalous double anterior spinal artery with selective disruption after traction, or from direct trauma to its feeding radiculomedullary artery at the time of hook placement on the right, the extent and location of the infarct being determined by the atavistic local arterial anatomy. Finally, such an injury may conceivably arise from venous infarction due to rupture of the thin ipsilateral spinal vein.

\section{ACKNOWLEDGEMENT}

Dr. R. Dewar, Orthopedic Surgeon, referred this patient.

\section{REFERENCES}

1. Wynne-Davis R. Familial (idiopathic) scoliosis: A family survey. J Bone Joint Surg (Br) 1968; 50: 24-3.

2. Winter RB. Adolescent idiopathic scoliosis. N Engl J Med 1986; 314: $1379-80$

3. Kevin HA. Fundamentals and basic principles of scoliosis. Orthopaedic Review 1983; 12: 31-40.

4. MacEwen GD, Bunnell WP, Sriram K. Acute neurological complications in the treatment of scoliosis. J Bone Joint Surg (Am) 1975; 57-A: 404-8.

5. Wilber RG, Thompson GH, Shaffer JW, et al. Postoperative neurological deficits in segmental spinal instrumentation. J Bone Joint Surg (Br) 1984; 66: 1178-1187.

6. Nash CL, Brown RH. The intraoperative monitoring of spinal cord function: its growth and current status. Orthop Clin North Am 1979; 1: 919-926.

7. Lesser RP, Randzens P, Luders H, et al. Postoperative neurological deficits may occur despite unchanged intraoperative somatosensory evoked potentials. Ann Neurol 1986; 19: 22-25.

8. Bernard TN, Johnston CE, Roberts JM, et al. Late complications due to wire breakage in segmental spinal instrumentation. J Bone Joint Surg (Am) 1983; 65-A: 1339-1345.

9. Hilal SK, Keim HA. Selective spinal angiography in adolescent scoliosis. Radiology 1972; 102: 349-359.

10. Dolan EJ, Transfeldt EE, Tator $\mathrm{CH}$ et al. The effect of spinal distraction on regional spinal cord blood flow in cats. J Neurosurg 1980; 53: 756-764.

11. Wells CEC. Clinical aspects of spinovascular disease. Proc Roy Soc Med 1966; 59: 790-795. 